\title{
EVALUACIÓN DEL RIESGO DE TOXICIDAD CRÓNICA POR EXPOSICIÓN A CADMIO EN RATAS (Rattus norvegicus)
}

\author{
Risk assessment of chronic toxicity for exposure to cadmium in rats (Rattus norvegicus)
}

\author{
${ }^{1}$ Mesías M. García, ${ }^{2}$ Eloisa M. Hernández \\ ${ }^{1}$ Instituto de Investigación de Recursos Naturales y Terapéuticos. ${ }^{2}$ Instituto de Investigación en Bioquímica Microbiología y \\ Biotecnología. Facultad de Farmacia y Bioquímica, Universidad Nacional Mayor de San Marcos
}

\section{RESUMEN}

El cadmio es un mineral que por su amplia utilización a nivel industrial puede ingresar a la cadena alimentaria y presentar bioacumulación en los diferentes sistemas alimentarios y en el organismo humano. El objetivo fue evaluar el riesgo de toxicidad crónica, caracterizando posibles efectos teratogénicos y signos clínicos de toxicidad en ratas albinas (Rattus norvegicus) de la cepa Holtzman. Para el diseño experimental se adecuaron las Guías de la Organización para la Cooperación Económica y Desarrollo (OECD). Se ensayaron dos niveles de ingestas de nitrato de cadmio: 40 y $8 \mathrm{o} \mu \mathrm{g} \mathrm{Cd} / \mathrm{día}$, administrados con el agua de bebida durante 180 días, con el respectivo grupo control. Los signos y efectos teratogénicos se determinaron por observación directa mediante evaluación macroscópica y registros fotográficos. Las ratas madre, durante la gestación, y sus crías, desde el nacimiento hasta 120 días, fueron observadas en sus conductas. En los tres grupos, las crías al nacer se diferenciaron por el peso promedio \pm desv. est. (grupo control: 7,22 \pm 1,1; grupo $40 \mu \mathrm{g} \mathrm{Cd} /$ día: 5,86 \pm o,45; y grupo $80 \mu \mathrm{g} \mathrm{Cd} /$ día: 5,30 $\pm 0,46 \mathrm{~g}$ ), por el tamaño corporal, color de la piel presentando signos hemorrágicos. El saco embrionario a los 18 días de la gestación, fue de color rosado el grupo control y varió a rosado violáceo y a rojo vino en los grupos ensayo expuestos a las ingestas diarias de cadmio. En las crías la acondroplasia fue manifiesta desde que nacieron y durante todo el período experimental. Se concluye que el cadmio produce efectos teratogénicos y tóxicos con daño estructural y funcional en hígado, útero y órganos anexos y en la vascularización, en ratas albinas adultas expuestas a ingestas crónicas de este elemento.

Palabras clave: Cadmio, toxicidad crónica, efecto teratogénico, Rattus norvegicus.

\section{SUMMARY}

Cadmium beacause its widespread use at industrial level can enter to food chain and cause bioaccumulation in different feed systems and in the human body. The objective was to assess the risk of chronic toxicity, characterizing potential teratogenic effects and clinical signs of toxicity in albino rats (Rattus norvegicus) of the Holtzman strain. To Guides experimental design of the Organization for Economic Cooperation and Development (OECD) were adapted. Two levels of cadmium nitrate intakes were tested: 40 and $80 \mu \mathrm{g} \mathrm{Cd}$ /day, administered with drinking water for 120 days; with the respective control group. Mothers rats, during pregnancy, and their offspring, from birth to 120 days, were observed in their behaviors The pups were differenced by birth weight (control group: 7,22 $\pm 1,1 ; 40 \mu \mathrm{g} \mathrm{Cd}$ /day group: 5,86 $\pm 0,45$; and $80 \mu \mathrm{g} \mathrm{Cd}$ /day group: 5,30 $\pm 0,46 \mathrm{~g}$ ), by body size, skin color with bleeding signs. The embryo sac at 18 days of gestation, showed differences in color, this was pink in the control group and ranged red wine in groups exposed to daily intakes of cadmium trials. In pups achondroplasia was manifested during the experimental period. Was concluded that cadmium produces teratogenic and toxic effects with structural and functional damage in liver, uterus and annex and vascularization in adult albino rats exposed to chronic intake of the mineral.

Keywords: Cadmium, chronic toxicity, teratogenic effect, Rattus norvegicus.

\section{INTRODUCCIÓN}

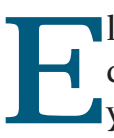

1 cadmio está distribuido en toda la corteza terrestre asociado a plomo, cobre y zinc. Actualmente debido a su amplia utilización en la industria puede convertirse en uno de los contaminantes del ambiente.

Existe transposición del cadmio en los ecosistemas desde el suelo hacia los organismos acuáticos y terrestres. Esto genera bioacumulación en moluscos, peces, aves marinas y mamíferos, quienes presentan incrementos de dicho elemento en hígado y riñones, posiblemente por sus hábitos alimentarios ${ }^{(1)}$.

Alimentos tales como hígado, riñones, crustáceos, moluscos, algunos champiñones y setas, pueden contener elevadas concentraciones de cadmio. En productos lácteos, huevos, pescados y carnes, la concentración hallada es menor que en vegetales ${ }^{(2)}$.

Hay evidencia de que los cereales lo acumulan a partir de suelos con fertilizantes. Se ha encontrado que la avena cultivada en suelos sin fertilizar contenía $0,02 \mu \mathrm{g} / \mathrm{g}$, mientras que la cultivada en suelos con 
fertilizantes presentó $0,28 \mu \mathrm{g} / \mathrm{g}{ }^{(3)}$. En el arroz cultivado en suelos contaminados se encontró más de $1 \mu \mathrm{g} / \mathrm{g}$ y en el que fue cultivado en la región donde ocurrió la epidemia de itai-itai las concentraciones variaron entre 1,72 a 4,12 $\mu \mathrm{g} / \mathrm{g}^{(3)}$.

Sus efectos tóxicos fueron descritos a consecuencia de la contaminación de animales por cadmio, debido a una mina cercana a la ciudad de Toyama, Japón. Estudios de correlación entre la contaminación del ambiente por cadmio y la salud han evidenciado que las concentraciones en agua, aire y alimentos fueron más altas en los lugares donde se registraron episodios de intoxicación en la población.

Es importante señalar que desde el año 1999, el cadmio ha sido clasificado como carcinógeno humano de categoría 1, por la Agencia Internacional para la Investigación del Cáncer ${ }^{(2,4)}$. Al presente, existe interés sobre los efectos de este mineral en la salud humana tales como disfunción y daño renal, hipertensión, carcinogénesis, mutagénesis y alteraciones en los huesos, en la función respiratoria, reproductiva e inmunitaria.

El objetivo fue caracterizar posibles teratogénesis y alteraciones histopatológicas en ratas con ingesta crónica de cadmio.

\section{MATERIALES Y MÉTODOS}

\section{Material biológico:}

Se adquirieron 30 ratas albinas adultas especie Rattus norvegicus cepa Holtzman, del Centro Nacional de Control de Productos Biológicos del Instituto Nacional de Salud, para los ensayos preliminares y la determinación de los efectos, teratogénicos, anatomomorfológicos e histopatológicos.

\section{Equipos:}

Balanza analítica de 250 g de capacidad.

Microscopio Bestec Scientific.

Baño maría Memmert $37^{\circ} \mathrm{C}$.

Microcentrífuga Mod KHT-4oo.

\section{Reactivos:}

Solución estándar de nitrato de cadmio, batch HCT 784320, $1000 \mathrm{mg} \mathrm{Cd/L.}$

\section{Métodos:}

La evaluación de la toxicidad crónica se realizó mediante el estudio de los efectos teratogénicos y de los efectos anátomo-morfológicos durante 180 días.

Las pruebas de teratogenicidad se realizaron adecuando las Guías de la Organización para la Cooperación Económica y Desarrollo (OECD) ${ }^{(5,6,7)}$. Los ensayos y determinaciones analíticas se llevaron a cabo en el Centro de Información y Control Toxicológico (CICOTOX) de la Facultad de Farmacia y Bioquímica, Universidad Nacional Mayor de San Marcos.

Las 30 ratas tuvieron un período de aclimatación de una semana en el bioterio. En 12 de ellas se condujo 
un ensayo previo para conocer los volúmenes de ingesta de agua y poder dosificar el cadmio.

La determinación de los efectos teratogénicos y anátomo-morfológicos se realizó con 18 ratas distribuidas en tres grupos de (3 machos y 3 hembras), como sigue:

Grupo 1. Control, reciben alimento habitual y agua de bebida exenta de cadmio.

Grupo 2. Reciben alimento habitual y dosis de $40 \mu \mathrm{g}$ Cd/día en el agua de bebida.

Grupo 3. Reciben alimento habitual y dosis de $80 \mu \mathrm{g}$ Cd/día en el agua de bebida.

Cada rata fue identificada e individualizada mediante colores y una marca externa particular para cada una. Fueron mantenidas en jaulas de metal galvanizado, con comedero y bebedero incorporados.

Apareo e identificación del estado de gravidez: Las ratas hembra con 90 días de edad o $180 \mathrm{~g}$ de peso, fueron apareadas mediante el sistema de lotes polígamos ${ }^{(5)}$.

El estado de gravidez se identificó por:

- La presencia del tapón copulatorio, mediante observación directa.

- Monitoreo citológico vaginal, mediante extracción de muestra vaginal y observación microscópica de la presencia de espermatozoides.

- Incremento de peso, desarrollo abdominal y de las mamas, por registro diario de peso y observación directa del abalonamiento abdominal y del crecimiento de las mamas.

Observación durante la gestación: Se observaron parámetros de actividades espontáneas, reactividad, pasividad, exaltación por los ruidos, tono corporal y muscular, color de la oreja, frecuencia cardíaca, frecuencia respiratoria, sensibilidad al dolor, convulsiones. El peso de cada rata se registró desde el inicio del apareamiento hasta el final de la gestación.
Observación pre y post natal: para conocer datos sobre abortos, mortalidad neonatal, porcentaje de fertilidad, pesos promedio al nacer. Se registró el número de crías por parto.

Evaluación de la teratogenicidad: Se realizó mediante la observación de ratas en gestación y de sus crías desde el nacimiento hasta los 120 días, en que alcanzan la edad adulta. En las crías se registraron datos sobre el número, peso, crecimiento (acondroplasia) y hemangiomas.

Evaluación de la toxicidad: Se realizó mediante la observación macroscópica de signos clínicos de intoxicación durante la gestación y mediante la observación anátomo-morfológica de los órganos internos.

Para la evaluación macroscópica de los órganos internos, se sacrificó una rata de cada uno de los grupos a los 18 días de gestación. Se emplearon los registros fotográficos para evaluar cambios en hígado, riñones, testículos, ovario, útero y órganos anexos, así como en la vascularización.

En la evaluación, de las ratas gestantes, también se registraron los cambios en el comportamiento y alteraciones externas como: respuestas somáticas, coloración de piel, ojos y orejas.

Análisis estadístico: Se aplicó estadística descriptiva y cuando fue necesario los modelos matemáticos $t$ de Student, Tukey y Anova.

\section{RESULTADOS}

\section{Teratogenicidad}

En la tabla 1 se presenta la ganancia de peso de las ratas durante la gestación y el número y peso de las crías al nacer y a los 21 días de edad.

Los promedios de ganancia de peso, fueron: 95,8 g; $59 \mathrm{~g}$ y $42 \mathrm{~g}$, en los grupos 1,2 y 3 , respectivamente.

Tabla 2. Signos clínicos de toxicidad en órganos de ratas con ingesta crónicas de cadmio vs. ratas del grupo control.

\begin{tabular}{|c|c|c|c|}
\hline $\begin{array}{l}\text { Órganos internos y } \\
\text { vascularización }\end{array}$ & Grupo Control & Grupo $40 \mu \mathrm{g}$ Cd/día & Grupo $80 \mu \mathrm{g}$ Cd/día \\
\hline Hígado & Tamaño y color normales & Hepatomegalia & Hepatomegalia y signos de hemorragia \\
\hline \multirow[t]{3}{*}{ Útero } & Embrión de color rosado & $\begin{array}{l}\text { Embrión color } \\
\text { rojo vino }\end{array}$ & $\begin{array}{l}\text { Embrión color rojo vino y con manchas de } \\
\text { color negro }\end{array}$ \\
\hline & $\begin{array}{l}\text { Aspecto normal de vasos } \\
\text { fetales }\end{array}$ & $\begin{array}{l}\text { Variación en el color de los } \\
\text { vasos fetales }\end{array}$ & Variación en el color de los vasos fetales \\
\hline & Envolturas del embrión limpias & Envolturas de embrión opacas & $\begin{array}{c}\text { Envolturas del embrión con alto contenido } \\
\text { de cadmio }\end{array}$ \\
\hline $\begin{array}{l}\text { Intestino y órganos } \\
\text { anexos }\end{array}$ & Color rosado & Color gris & Color gris \\
\hline Vascularización & $\begin{array}{l}\text { Normal } \\
\text { Sin focos hemorrágicos }\end{array}$ & Envoltura anormal & $\begin{array}{c}\text { Aumento anormal } \\
\text { Focos hemorrágicos } \\
\text { Engrosamiento de venas y arterias }\end{array}$ \\
\hline
\end{tabular}


El número total de crías fue de 37,31 y 30 , respectivamente; de las cuales nacieron vivas el $100 \%$; $93,5 \%$ y $54 \%$ en los respectivos grupos.

Lospesos delas crías a los 21 días de edad $(57,6 \mathrm{~g} ; 38,9 \mathrm{gy}$ $36,8 \mathrm{~g}$ ), evidenciaron gran diferencia entre los dos grupos expuestos y el control.

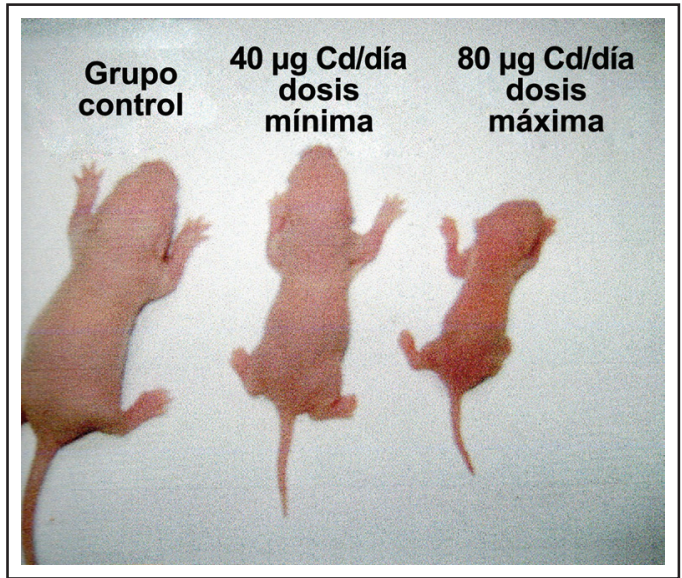

Figura 1. Tamaño corporal de las crías al nacer según dosis de cadmio ingerida.

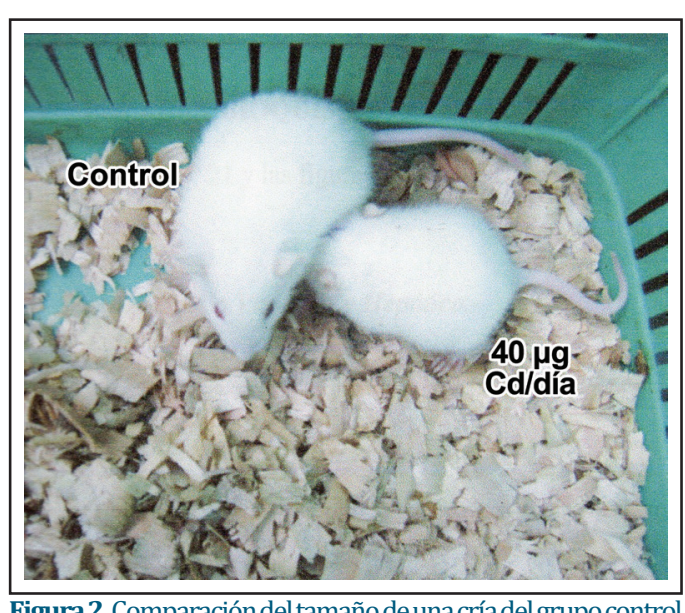

Figura 2. Comparación del tamaño de una cría del grupo control (C), frente a otra del grupo de dosis mínima $(40 \mu \mathrm{g} C \mathrm{~d} /$ día $)$.

Entre las ratas que ingerían $80 \mu \mathrm{g} C \mathrm{C} /$ día, de las 20 crías que nacieron vivas, 5 murieron antes de los 21 días.

En la figura 1, se presenta el tamaño corporal de las crías -al nacer- de acuerdo a la dosis de Cadmio ingerido. En la figura 2 se comparan los tamaños de un animal del grupo control frente a otro del grupo de dosis mínima.

Evaluación de los órganos internos: Se observa, en la comparación entre los registros fotográficos del saco embrionario de animales sacrificados a los 18 días de gravidez, pertenecientes a los tres grupos estudiados, que en las ratas del grupo control, este tejido mostró características normales, mientras que a mayor dosis de cadmio, el color varió hasta un tono rojo vino oscuro (figuras 3, 4 y 5).

Presencia de hemangiomas: Las crías recién nacidas del grupo control se distinguen por su mayor tamaño y la coloración rosada de su piel. Las provenientes de madres con ingesta de $40 \mu \mathrm{g} \mathrm{Cd} /$ día,

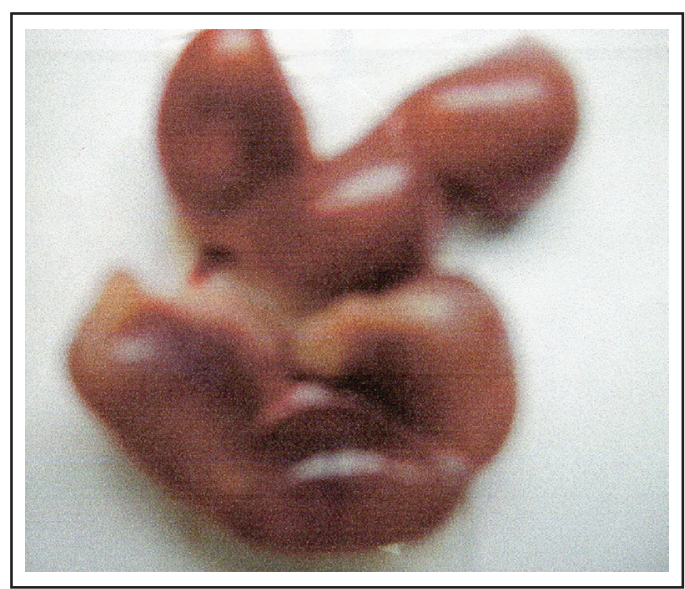

Figura 3. Saco embrionario rata control.

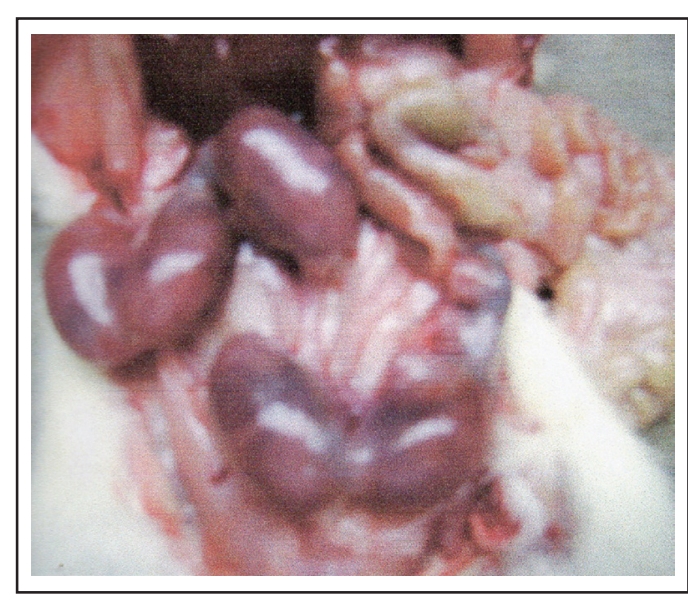

Figura 4. Saco embrionario de rata expuesta a 40 g Cd/día. fueron más pequeñas con la piel de color violáceo. Las crías de madres con ingesta de $80 \mu \mathrm{g}$ de $\mathrm{Cd} /$ día, nacieron cianóticas y fueron de menor tamaño (figuras 6, 7 y 8).

\section{Toxicidad}

En la tabla 2 se presentan los signos clínicos de toxicidad observados en órganos internos y en la vascularización. La ingesta de dosis de $80 \mu \mathrm{g} \mathrm{Cd} /$ día tuvo mayor impacto sobre estos tejidos.

\section{DISCUSIÓN}

La exposición crónica a cadmio altera el metabolismo de los minerales esenciales en el hígado causando disminución de fierro y de selenio. Las carencias nutricionales provocan una mayor absorción de cadmio. El hallazgo de acondroplasia (disminución del tamaño corporal) podría deberse a la acción de este metal sobre la hormona de crecimiento, lo que ha sido citado por Piasek y Laskey ${ }^{(8)}$.

\section{En} el presente estudio, este efecto tóxico se manifestó desde el nacimiento, continuó hacia los 21 días de edad y perduró durante los 180 días que duró el experimento. Las diferencias observadas entre 


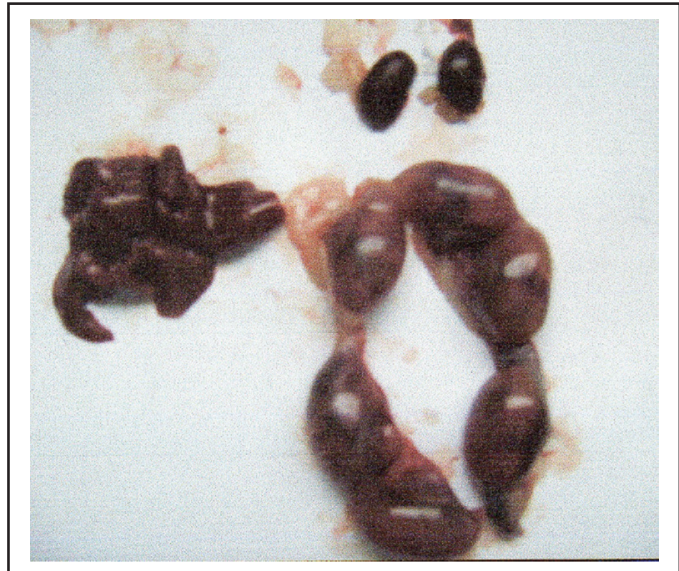

Figura 5. Saco embrionario de rata expuesta a 80 g Cd/día.

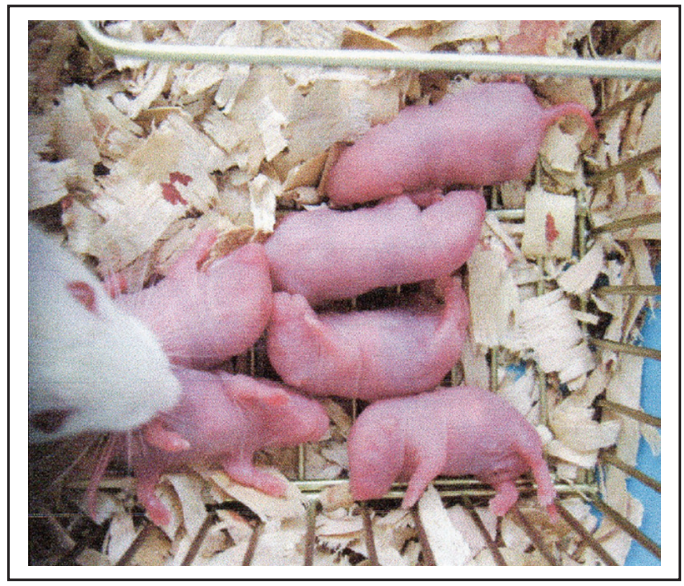

Figura 7. Crías de rata grupo $40 \mu \mathrm{g} \mathrm{Cd/día.}$

control y las expuestas a ingesta (40 $\mu \mathrm{g} \mathrm{Cd} /$ día $)$, fueron significativamente altas $(p<0,004)$.

La alteración de la reproducción evidenciada por la disminución del número de crías también fue manifiesta en los dos grupos con ingesta de cadmio.

Los efectos del cadmio sobre el saco embrionario, no han sido tipificados como teratogénicos. En la presente investigación se observaron cambios que afectaron el color y la estructura de este tejido en ratas con 18 días de gestación.

El efecto teratogénico evidenciado por la presencia de signos hemorrágicos (hemangiomas), es análogo a lo registrado por otros autores en ratones, en los que la ingesta de cadmio a dosis de 50, 100 y 150 $\mu \mathrm{g} / \mathrm{kg}$ de peso corporal, produjo degeneración en la corteza y vasodilatación sanguínea con tendencia a la hemorragia ${ }^{(9)}$.

Con relación a los signos clínicos de toxicidad, el daño hepático, traducido por vacuolización
CIENCIA E INVESTIGACIÓN 2014; 17(2): 74-79

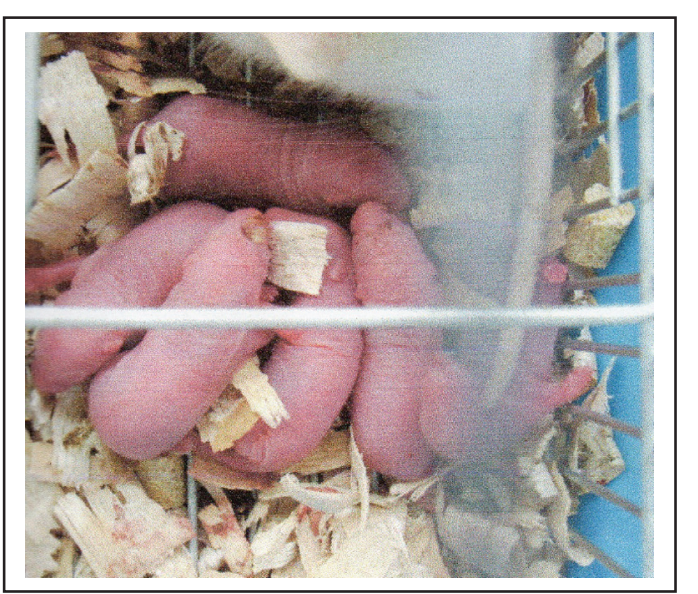

Figura 6. Crías de rata grupo control.

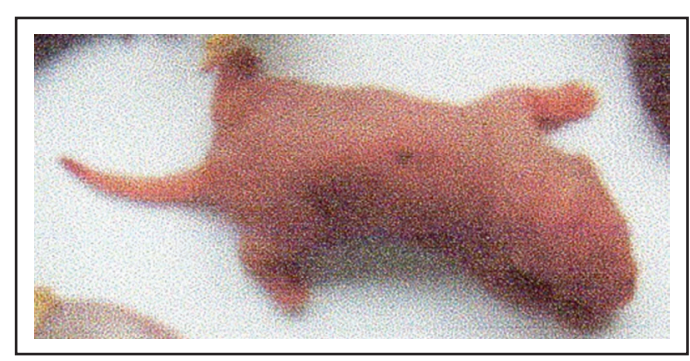

los pesos de las ratas del grupo

4

a
40 y $80 \mu \mathrm{g} \mathrm{Cd} /$ día produce alteraciones estructurales
y funcionales en hígado, útero, intestino y órganos
anexos de ratas.

La ingesta de $80 \mu \mathrm{g}$ Cd/día origina efectos teratogénicos en ratas en estado de gestación, toxicidad reproductiva, bajo peso, bajo porcentaje de fertilidad, reducción del número de crías por camada, elevada mortalidad, daño estructural y funcional; y en las crías, al nacer, produce hemangiomas y acondroplasia.

\section{REFERENCIAS BIBLIOGRÁFICAS}

1. Organización Internacional del Trabajo, OIT. Enciclopedia de salud y seguridad en el trabajo. $3^{\text {ra }}$ ed. Ministerio de Trabajo. Madrid, 2001.

2. Galvão L, Corey G. Cadmio. Serie vigilancia 4. Centro Panamericano de Ecología Humana y Salud. OPS/OMS. Mexico, Metepec, 1987.

3. Valle P. Toxicología de Alimentos. Centro Nacional de 
Salud Ambiental. Instituto Nacional de Salud Pública. México DF, 2000.

4. Waalkes M. Cadmiun carcinogenesis in review. J Inorg Biochem 2000, 79(1-4): 241-4.

5. OECD Guideline for Testing of Chemicals Organization for Economic Cooperation and Development. Guide $\mathrm{N}^{\circ}$ 414, 2001.

6. OECD Guideline for Testing of Chemicals Organization for Economic Cooperation and Development. Guide $N^{\circ}$ $415,1983$.

7. OECD Guideline for Testing of Chemicals Organization for Economic Cooperation and Development. Guide $N^{\circ}$ 416, 2001.

8. Piasek M, Laskey JW. Effect of in vitro cadmium exposure on ovarian steroidogenesis in rats. J Appl Toxicol 1999; 19(3): 211-7.

9. Lapa B. Evaluación del efecto teratogénico del Cadmio en ratas albinas grávidas. [Tesis para optar el título de Químico Farmacéutico] Facultad de Farmacia y Bioquímica. Universidad Nacional Mayor de San Marcos. Lima, 1999.

10. Iglesias A, Martin R, Santa María L. Inducción de adenomas de células de Leydig en testículo de ratas expueastas a cloruro de cadmio. Urología integrada y de Investigación 2002; (7): 351-70.

Manuscrito recibido el: 19/o8/14

Aceptado para su publicación el: 06/10/14

\section{Correspondencia:}

Nombre: Mesías Moisés García Ortiz

Direccion: $\quad$ Jr. Puno 1002 - Lima o1

Email: mgarciao@unmsm.edu.pe 